\title{
Title: Evaluation of the concordance of immunological biomarkers between core biopsy and corresponding resection specimen in ER/PR negative breast cancer
}

Hanne Vos ${ }^{1,2}$ MSc, Kathleen Lambein ${ }^{1,2}$ MD; PhD, Ines Nevelsteen ${ }^{1,2}$ MD; PhD, Giuseppe Floris ${ }^{2,3}$ MD; PhD, Annouschka Laenen ${ }^{4}$ PhD, Patrick Neven ${ }^{2,5}$ MD; PhD, Hans Wildiers ${ }^{2,6}$ MD; PhD, Ann Smeets ${ }^{1,2}$ MD; PhD.

1) Department of surgical oncology, University Hospitals Leuven, KULeuven

2) Multidisciplinary breast centre, University Hospitals Leuven

3) Translational cell \& tissue research, University Hospitals Leuven, KULeuven

4) Leuven Biostatistics and Statistical Bioinformatics Centre (L-BioStat)

5) Department of gynaecology and obstetrics, University Hospitals Leuven, KULeuven

6) Department of general medical oncology, University Hospitals Leuven, KULeuven

Background: There is an urgent need for biomarkers in breast cancer. Clinical trials allow to compare the expression of immunological biomarker candidates before and after neo-adjuvant treatment. However, evidence for a concordance between core and resection specimen for these immunological markers is still lacking.

Material and methods: Core biopsy and matching resection specimen were analysed for 35 patients who underwent primary surgery and 15 patients who had neo-adjuvant chemotherapy (NACT) for an early ER/PR negative invasive adenocarcinoma of at least $2 \mathrm{~cm}$. Haematoxylineosin staining was performed to determine the percentage of tumour-infiltrating lymphocytes (TILs). The expression of CD3, CD4, CD8, CD68, CD73, FoxP3, PD-1 and Ki67 was assessed semi-quantitatively using immunohistochemistry. Statistical analysis was performed to calculate the concordance and intraclass correlation coefficient (ICC) between core biopsy and resection specimen.

\section{Results:}

- Upfront surgery group: A positive association was found between core biopsy and resection specimen for the following variables: TILs, CD3, CD8 and Ki67. The ICC showed an excellent and good agreement for Ki67 and TILs scores respectively, and a poor agreement for CD3 and CD8 scores. No association was found for CD4, CD68, CD73, FoxP3 and PD-1.

- NACT group: A positive association was only found between core biopsy and resection specimen for Ki67 and the ICC showed a fair agreement. No association was found for TILs, CD3, CD4, CD8, CD68, CD73, FoxP3 and PD-1.

Conclusions: Ki67 and TILs show a good concordance between the core biopsy and the corresponding resection specimen in the upfront surgery group. Concordance is limited for the other tested immunological markers. The agreement was poor in the NACT group as expected, with chemotherapy as plausible confounding factor. These findings shed new light on the predictive or prognostic use of immunological biomarkers measured in core biopsies. Additional data are required for validation. 